\title{
Influence of Correlated Disorder Potentials on the Levitation of Current Carrying States in the Quantum Hall Effect
}

\author{
Th. Koschny and L. Schweitzer* \\ Physikalisch-Technische Bundesanstalt, Bundesallee 100, 38116 Braunschweig, Germany
}

\begin{abstract}
The disorder driven quantum Hall to insulator transition is investigated for a two-dimensional lattice system. We consider a Gaussian correlated random potential, study the behaviour of the current carrying states and trace their energetical position when the disorder strength is increased. Our results qualitatively resemble those obtained previously for exponentially correlated disorder potentials. We find both the downward movement of the anti-Chern states as well as the floating up of the Chern states across the Landau gap which is sometimes masked by the global broadening of the tight binding band.
\end{abstract}

Keywords: Quantum Hall to insulator transition, correlated random potentials, current carrying states

\section{Introduction}

The disorder driven quantum Hall to insulator transition once again became a topic of active research recently. The theoretical activities undertaken over the past years resulted in a controversial debate concerning the fate of the current carrying states [1, 2, 3, 4, 5, 6, 7]. Likewise, the experimental results reported all along remain inconclusive [8, 9, 10, 11, 12, 13, 14, 15]. In numerical studies using uncorrelated random disorder potentials, it was found that in contrast to the continuum model, where the critical states are known to float up in energy [16, 17, 18], the current carrying states are annihilated by anti-Chern states originating from the band centre in the lattice model [2, 4,6$]$. It has also been claimed [4, 5] that such a scenario should be able to explain the direct $\mathrm{QH}$ to insulator transitions from higher Hall plateaus with filling factor $\nu>2$, which apparently have been observed in several experiments. However, according to the proposed global phase diagram [19], which is based on the levitation picture, such transitions should not be allowed.
In a recent paper we have shown [20] that the lattice model can exhibit similar properties as the continuum model if long range correlated random disorder potentials are considered. Using exponentially correlated random potentials to model the intrinsic scattering potentials and other imperfections that may influence the movement of the charge carriers in real 2DEGs, we found that the floating up in energy can already be observed for correlation lengths larger than about half the lattice constant. In particular, with increasing disorder the floating up of the critical energy across the Landau gap without merging has been demonstrated. The latter is in contrast to the results of a numerical study [21] where Gaussian correlated disorder potentials were used. In the present paper we investigate whether the observed discrepancy is due to the differences in the correlated disorder potentials considered. Therefore, in the following we also use Gaussian correlated disorder potentials and calculate numerically the disorder dependence of the density of states and investigate the finite size scaling of the localisation length from which the energetical position of the current carrying states can be extracted.

\footnotetext{
${ }^{*}$ Corresponding author. Fax: +495315928106; email: Ludwig.Schweitzer@ptb.de.
} 


\section{Model and Method}

The QHE system is described by a disordered two-dimensional single band lattice model [22, 20], $H=\sum_{m} w_{m} c_{m}^{\dagger} c_{m}+$ $\sum_{<m n>}\left(V_{n m} c_{m}^{\dagger} c_{n}+V_{m n} c_{n}^{\dagger} c_{m}\right)$, where $c_{m}, c_{m}^{\dagger}$ are the creation and annihilation operators of a particle at site $m$, respectively. The perpendicular magnetic field is incorporated via the complex phases of the transfer terms, $V_{m n}=$ $V \exp \left(i b_{m n}\right)$, between neighbouring sites $m, n$ on the lattice. In the Landau gauge, $b_{m n}=$ $\pm 2 \pi(p / q)\left(\vec{r}_{m} \cdot \vec{e}_{y}\right) / a$, if $\vec{r}_{n}=\vec{r}_{m} \pm a \vec{e}_{x}$, and $b_{m n}=0$ else, where $\vec{r}_{m}$ is the position of site $m$ and $\vec{e}_{x}, \vec{e}_{y}$ are unit vectors pointing in the $x$ and $y$ directions. We choose $p / q=1 / 8$ flux quanta $h / e$ per plaquette $a \times a$, where $a$ is the lattice constant. The Gaussian correlated disorder potentials $w_{m}$ are generated from uncorrelated random variables $\varepsilon_{n}$ associated with each lattice site $n$ by local averaging using a Gaussian weighting function, $w_{m}=$ $1 / N \sum_{n} \varepsilon_{n} \exp \left(-|m-n|^{2} / \eta^{2}\right)$, with correlation length $\eta$ and some normalisation factor $N$. This leads to a spatial correlation function decaying qualitatively like $\left\langle w_{m} w_{n}\right\rangle \sim$ $\exp \left(-|m-n|^{2} / 2 \eta^{2}\right)$. The probability density of the $\varepsilon_{n}$ is taken to be $P\left(\varepsilon_{n}\right)=1 /(2 W)$ in the range $-W \leq \varepsilon_{n} \leq W$, and zero else.

The density of states $\rho(E, W)$ is calculated from the distribution of energy eigenvalues which are obtained by direct diagonalisation of square systems averaged over 60 realisations of the correlated disorder potentials. The exponential localisation length $\lambda_{M}^{-1}(E, W)=$ $-\lim _{L \rightarrow \infty}(2 L)^{-1} \ln \operatorname{Tr}\left\{\left|G_{1 L}\right|^{2}\right\}$ is calculated numerically by means of a recursive Green function method [23, 22]. $G \equiv(E-H+i \gamma)^{-1}$ is the total Green function and $G_{1 L}$ is defined on the subspace of the first and last columns on the lattice. The system's length and width are $L$ and $M$, respectively. Both, the density of states and the localisation length were computed for various system sizes, disorder strengths, and correlation lengths of the dis-

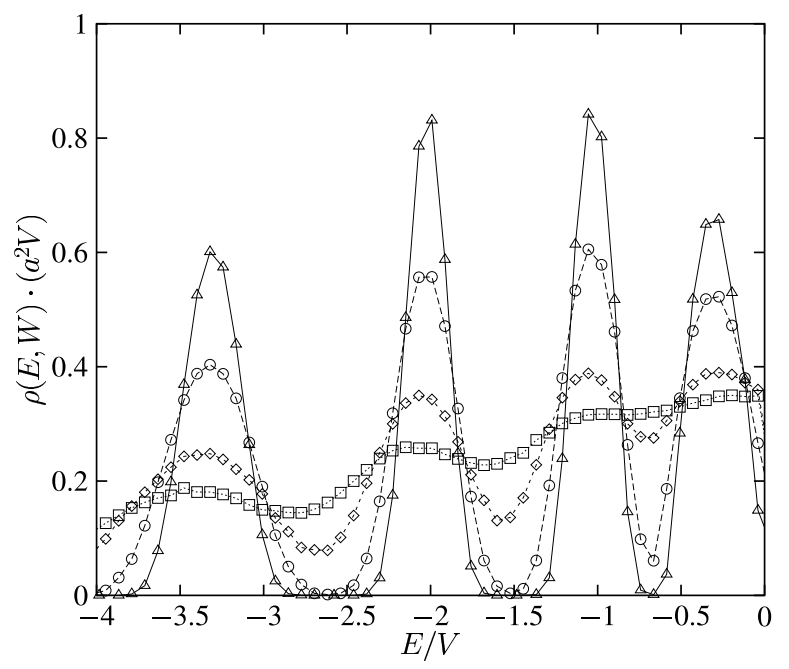

Fig. 1. The density of states $\rho(E, W)$ versus energy for disorder strengths $W / V=1.0,1.5,2.5$, and 3.5. The system size is $M / a=48$ and the lines simply connect the calculated data points.

order potentials. In what follows, we restrict the presentation of our results to those data obtained for correlation parameter $\eta / a=1.0$. The same value has been utilised in Ref. [21].

\section{Results and Discussion}

The movement of the energetic position of the current carrying states induced by a change of the disorder strength is obscured by the global broadening of the tight binding band with increasing disorder. To distinguish this effect, which in turn shifts the Landau bands outwards, from the true floating of the critical states, the density of states $\rho(E)$ is calculated first for several disorder strength in the range $0.5 \leq W / V \leq 5.0$. As an example, the result of $\rho(E)$ for disorder strength $W / V=$ 1.0, 1.5, 2.5, and 3.5 is plotted in Fig. 1. Due to the symmetry with respect to $E / V=0$, only the lower half of the total band is shown. With increasing disorder the sub-bands broaden and shift slightly outwards. The effect is stronger near the band edge. Similar curves were obtained for further disorder strengths. The position of the sub-band peaks were extracted from a curve-fitting procedure using Gaussian 


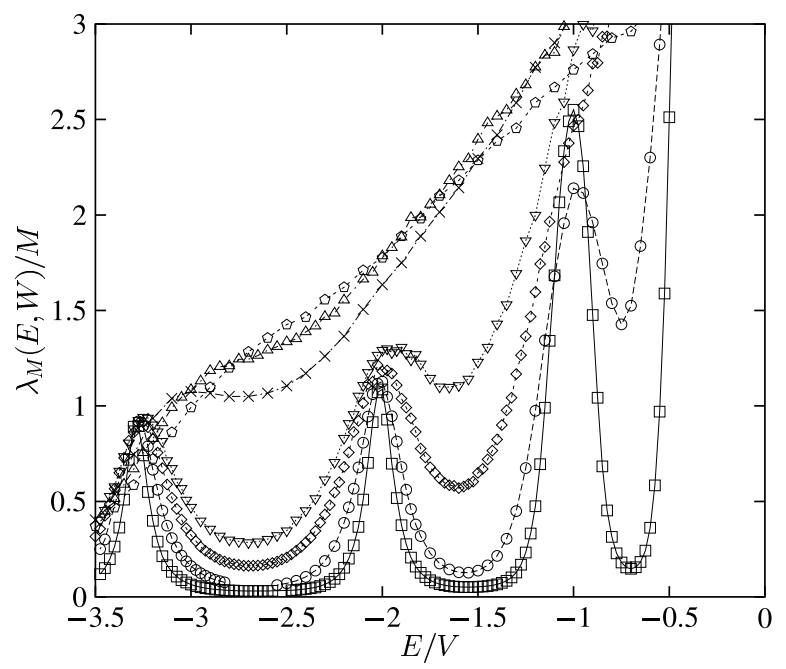

Fig. 2. The energy dependence of the normalised localisation length $\lambda_{M}(E, W) / M$ for disorder strengths $W / V=1.5,2.5,3.5,4.0,5.5,6.0$, and 6.5 . The system length is at least $L / a=3 \cdot 10^{4}$ and the width $M / a=48$.

or power-like shapes for the particular Landau bands, exploiting the normalisation condition for each single band and tracing the fit parameters from lower to higher disorder.

The energy dependence of the normalised localisation length $\lambda_{M}(E, W) / M$ has been calculated for disorder strengths in the range $1.0 \leq W / V \leq 6.5$. Curves for $W / V=1.5$, $2.5,3.5,4.0,5.5,6.0$, and 6.5 are plotted in Fig. 2, whereby the data points shown are averages over 30 disorder realizations. The system width is $M / a=48$ and the length varies between $L / a=3 \cdot 10^{4}$ and $L / a=5 \cdot 10^{5}$ depending on the varying convergence for different energy and disorder strength. For fixed system width $M, \lambda_{M}(E, W) / M$ increases with increasing disorder strength. Associated with this increase is a shift of the peak position, which corresponds to the floating up of the current carrying state to higher energies.

In Fig. 3 the energy and disorder dependence of $\rho(E, W)$ and $\lambda_{M}(E, W) / M$ are combined. The position of the sub-band peaks of the density of states $(\times)$ is shown within the disorder-energy plane. With increasing disorder, the density of state peaks shift to lower energy. This movement is well fitted by the

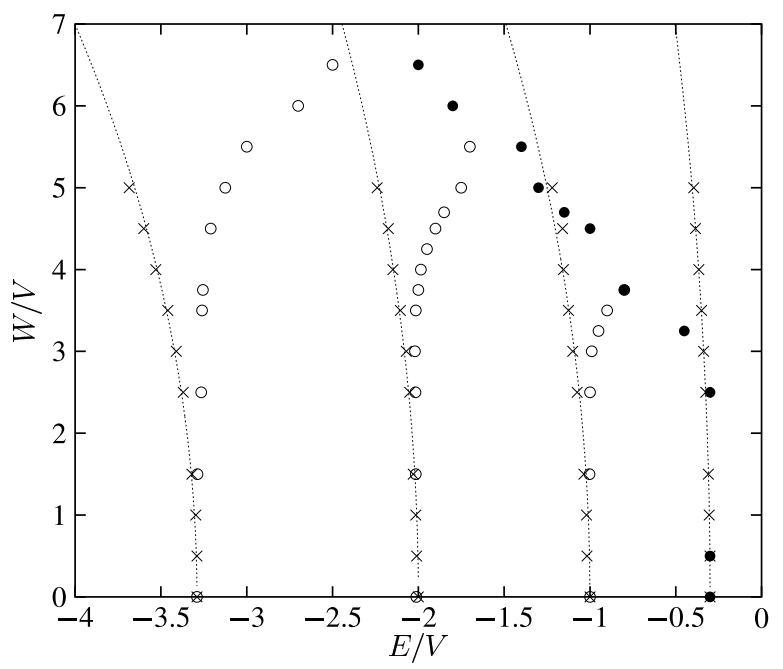

Fig. 3. The position of the sub-band peaks in the density of states $\rho(E, W)(\times)$ and the position of the normalised localisation length $\lambda_{M}(E, W) / M(O)$ within the disorder-energy plain. The anti-Chern states are depicted by (๑). The dotted line is an empirical fit $W_{p}(E)=A\left(E_{n}-E\right)^{1 / 2}$ to the peak position of the sub-bands.

empirical relation $W_{p}(E)=A\left(E_{n}-E\right)^{1 / 2}$ which corresponds to the observation that the bandwidth of the total band increases with disorder $\sim W^{2}$. The $E_{n}$ denote the energies of the sub-bands (Landau levels) for $W / V=0$. In addition to the sub-band peaks, the position of the current carrying states $(O)$ are plotted also in Fig. 3. The energies of the critical states were extracted from a finite size scaling analysis combined with a similar curvefitting procedure as used for the total density of states above. For each disorder strength $\lambda_{M}(E, W) / M$ decreases for localised states with increasing system size while it stays constant in the thermodynamic limit only at those energies that correspond to the current carrying states.

For small disorder $W$ both the energy of the peaks in the density of states and the energy of the critical states coincide. However, with increasing disorder strength, the curves move apart. The floating of the energy of the critical states in the opposite direction as the subbands, almost across the lowest Landau gaps, 
is clearly seen. The downward movement of the anti-Chern state $(\bullet)$ can easily be followed until it is finally annihilated by the Chern state of the lowest sub-band at a disorder strength of $W / V \sim 7$. This also defines the cross-over from the quantum Hall liquid state to the insulator.

Comparing our results with those published previously for exponentially correlated disorder potentials [24, 20], we notice that the behaviour of the current carrying states is qualitatively similar. With increasing correlations the position where the anti-Chern and the last Chern state meet is shifted to higher energies. Therefore, it is to be expected that for stronger correlations only the floating up of the energy of the current carrying states is observed as in the continuum model. The apparent merging and subsequent joint floating of the current carrying states as reported [21] for similar Gaussian correlated disorder potentials was not observed in our investigation. However, this may look different for smaller magnetic field as applied in [21] because the distinction of clustered critical states becomes increasingly problematic in finite size studies.

In conclusion, the disorder driven quantum Hall to insulator transition in a lattice model with Gaussian correlated random potentials has been investigated. For a correlation length $\eta / a=1$ we have shown that the energy of the lowest current carrying state floats up in energy across the Landau gap when the disorder strength is increased. This is similar to the levitation scenario proposed for the continuum model. In view of these results, the proposed explanation [21] for the apparent direct transitions to the insulator from plateaus with $\nu>2$ as being due to the downward movement of the anti-Chern states is unlikely. We believe that the direct transitions reported in experiments are either unresolved single one by one transitions or have to be accounted for by electronelectron interactions.

\section{References}

[1] K. Yang and R. N. Bhatt, Phys. Rev. Lett. 76, (1996) 1316.

[2] D. Liu, X. Xie, and Q. Niu, Phys. Rev. Lett. 76, (1996) 975.

[3] X. C. Xie, D. Z. Liu, B. Sundaram, and Q. Niu, Phys. Rev. B 54, (1996) 4966.

[4] D. N. Sheng and Z. Y. Weng, Phys. Rev. Lett. 78, (1997) 318.

[5] D. N. Sheng and Z. Y. Weng, Phys. Rev. Lett. 80, (1998) 580.

[6] H. Potempa, A. Bäker, and L. Schweitzer, Physica B 256-258, (1998) 591.

[7] K. Yang and R. N. Bhatt, Phys. Rev. B 59, (1999) 8144.

[8] A. A. Shashkin, G. V. Kravchenko, and V. T. Dolgopolov, JETP Lett. 58, (1993) 220.

[9] H. W. Jiang, C. E. Johnson, K. L. Wang, and S. T. Hannahs, Phys. Rev. Lett. 71, (1993) 1439.

[10] T. K. Wang et al., Phys. Rev. Lett. 72, (1994) 709.

[11] S. V. Kravchenko, W. Mason, J. E. Furneaux, and V. M. Pudalov, Phys. Rev. Lett. 75, (1995) 910.

[12] I. Glozman, C. E. Johnson, and H. W. Jiang, Phys. Rev. Lett. 74, (1995) 594.

[13] D. Shahar et al., Phys. Rev. Lett. 79, (1997) 479.

[14] C. H. Lee, Y. H. Chang, Y. W. Suen, and H. H. Lin, Phys. Rev. B 58, (1998) 10629.

[15] M. Hilke et al., Phys. Rev. B 62, (2000) 6940.

[16] D. E. Khmelnitskii, Phys. Lett. 106A, (1984) 182.

[17] R. B. Laughlin, Phys. Rev. Lett 52, (1984) 2304.

[18] T. Ando, Journal of the Physical Society of Japan 53, (1984) 3126.

[19] S. Kivelson, D.-H. Lee, and S.-C. Zhang, Phys. Rev. B 46, (1992) 2223.

[20] T. Koschny, H. Potempa, and L. Schweitzer, Phys. Rev. Lett. 86, (2001) 3863.

[21] D. N. Sheng, Z. Y. Weng, and X. G. Wen, condmat/0003117 (2000) .

[22] L. Schweitzer, B. Kramer, and A. MacKinnon, J. Phys. C 17, (1984) 4111.

[23] A. MacKinnon and B. Kramer, Z. Phys. B 53, (1983) 1.

[24] H. Potempa and L. Schweitzer, Physica B 298, (2001) 52. 